\title{
Assessment of vitamin K status by fully automated IDS-iSYS InaKtif MGP
}

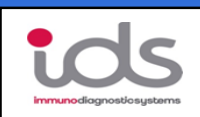

Dagmar Kasper ${ }^{1}$, Mhammed Bougoussa ${ }^{1}$, Cees Vermeer ${ }^{2}$

${ }^{1}$ IDS PIc, Boldon, UK

${ }^{2}$ VitaK, Maastricht University, The Netherlands

\section{Introduction}

Matrix Gla-protein (MGP) is a vitamin K-dependent protein acting as a potent inhibitor of soft tissue calcification, mainly in the vessel wall and cartilage [1]. During its synthesis MGP undergoes extensive posttranslational modification, i.e. serine phosphorylation and glutamate carboxylation (to form $\mathrm{Gla}$ ). Vascular vitamin $\mathrm{K}$ insufficiency is associated with the production of desphospho-uncarboxylated MGP (dp-ucMGP), which has no calcification inhibitory activity and which is set free in the circulation, where it can be quantified by assays based on the sandwich ELISA principle. Most data presently available in the literature are based on a handmade microtiter plate assay designed at VitaK laboratories [2]. Here we present a fully automated version of the same assay, which is produced by IDS: the InaKtif MGP assay.

\section{Materials \& Methods}

Antibodies used: Monoclonal antibodies against the phosphoserine domain (aa 3-15) and the Gla-domain (aa 35-49) in human MGP.

Assay principle: The IDS Automated Analyser IDS-iSYS InaKtif MGP assay uses both highly specific monoclonal antibodies. One biotinylated antibody is coupled to magnetic particles (MP). The second antibody is coupled to an acridinium ester derivative. $50 \mu \mathrm{l}$ of sample is incubated with $20 \mu \mathrm{L}$ of MP and the second antibody. After $60 \mathrm{~min}$ of incubation, followed by a washing step, triggers are added whereby the luminescence measured is directly proportional to the InaKtif MGP concentration present in the sample. The time to first result is 63 minutes. The throughput of the assay is 89 tests/hour.

Assay Diagram:

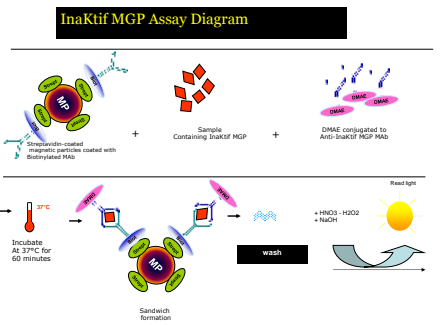

Figure 1: Schematic diagram of the IDS-iSYS InaKtif MGP assay. The signal produced is proportional to the InaKtif MGP concentration in the sample

Assay calibration. The IDS InaKtif MGP assay is calibrated against an in house standard prepared in horse serum using 2-point calibrations for master curve repositioning. The analytical range is $200-10000 \mathrm{pM}$.

Sample preparation: Blood (taken by venipuncture) should be collected in EDTA; plasma should be prepared and kept frozen at $-80^{\circ} \mathrm{C}$ until testing

\section{Results}

Sensitivity: Sensitivities LOB, LOD and LOQ were evaluated in accordance with CLSI EP17-A Guideline. LOB and LOD were determined respectively at 47 and $119 \mathrm{pM}$.

Precision: Precision was evaluated according to CLSI EP-5A Guideline. Five control plasma samples were assayed in duplicate twice a day for a minimum of 20 days.

\begin{tabular}{|c|c|c|c|c|c|c|}
\hline Control & $\mathbf{N}$ & Mean & \multicolumn{2}{|c|}{ Within - Run } & \multicolumn{2}{|c|}{ Total } \\
\hline & & $\boldsymbol{p M}$ & $\boldsymbol{S D}$ & $\boldsymbol{C V} \boldsymbol{H}$ & $\boldsymbol{S D}$ & $\boldsymbol{C V} \%$ \\
\hline $\mathbf{1}$ & 80 & 555 & 35 & 6.2 & 80 & 14.4 \\
\hline $\mathbf{2}$ & 80 & 1033 & 49 & 4.7 & 108 & 10.5 \\
\hline $\mathbf{3}$ & 80 & 2493 & 204 & 4.2 & 198 & 7.9 \\
\hline $\mathbf{4}$ & 80 & 4386 & 114 & 2.6 & 296 & 6.7 \\
\hline $\mathbf{5}$ & 80 & 7741 & 221 & 2.9 & 468 & 6.0 \\
\hline
\end{tabular}

Table 1: IDS-iSYS InaKtif MGP assay precision

Linearity: The linearity was realized according to the EP-6A CLSI recommendation. The IDS-iSYS InaKtif MGP assay shows a good linearity over the dynamic range (figure 2).

\begin{tabular}{llll} 
& Observed & Expected & O/E (\%) \\
\hline X1 & 0 & & \\
$0,875 \times 1+0,125 \times 2$ & 807 & 937 & 86 \\
$0,750 \times 1+0,250 \times 2$ & 1951 & 1873 & 104 \\
$0,625 \times 1+0,375 X 2$ & 3174 & 2810 & 113 \\
$0,500 \times 1+0,500 \times 2$ & 4121 & 3746 & 110 \\
$0,375 \times 1+0,625 X 2$ & 4875 & 4683 & 104 \\
$0,250 \times 1+0,750 \times 2$ & 5469 & 5620 & 97 \\
$0,125 \times 1+0,875 \times 2$ & 7024 & 6556 & 107 \\
X2 & 7670 & 7493 & 102 \\
\hline
\end{tabular}

Table 2: IDS-iSYS InaKtif MGP linearity over the dynamic range
Recovery: 3 serum samples were spiked with 2 different amounts of known MGP concentration. The recovery was determined by comparing recovered concentrations to the added concentrations. Data are shown in table 3.

\begin{tabular}{|c|c|c|c|c|}
\hline $\begin{array}{l}\text { InaKtif MGP } \\
\text { conc. (pM) }\end{array}$ & $\begin{array}{c}\text { MGP } \\
\text { added (pM) }\end{array}$ & $\begin{array}{c}\text { Measured } \\
\text { values (pM) }\end{array}$ & $\begin{array}{l}\text { Recovered } \\
\text { (pM) }\end{array}$ & $\begin{array}{c}\text { Recovery } \\
\text { (\%) }\end{array}$ \\
\hline 89 & 1383 & 1206 & 1117 & $86 \%$ \\
\hline 89 & 2677 & 2406 & 2317 & $90 \%$ \\
\hline 267 & 1561 & 1431 & 1164 & $90 \%$ \\
\hline 267 & 2855 & 2613 & 2346 & $91 \%$ \\
\hline 1269 & 2563 & 2672 & 1403 & $108 \%$ \\
\hline \multirow[t]{2}{*}{1269} & 3857 & 4041 & 2772 & $107 \%$ \\
\hline & & & Average & $95 \%$ \\
\hline
\end{tabular}

Table 3: Recovery results with IDS-iSYS InaKtif MGP assay

Correlation between InaKtif MGP assay and hand-made ELISA: The IDS-iSYS InaKtif MGP assay was compared with the VitaK homemade ELISA (figure 2). Linear regression was used for comparative data:

IDS-iSYS InaKtif MGP = $\mathbf{1 . 0 5 6}(\mathbf{x})+\mathbf{5 4 . 3 4}$ with a coefficient of correlation $\mathrm{R}=0.98$

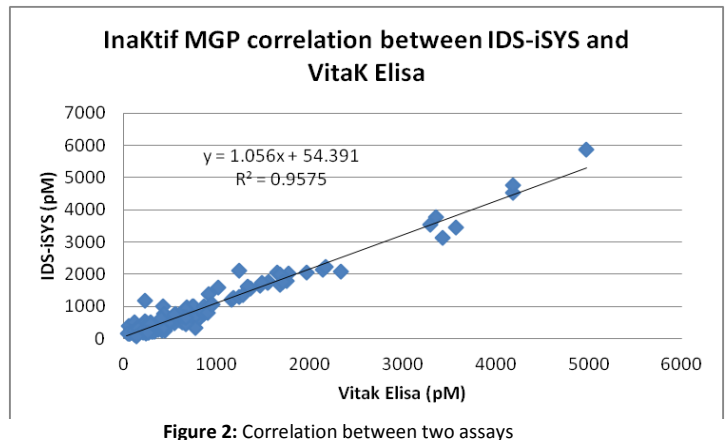

Vitamin K status The microtiter plate assay and the InaKtif MGP assay were measured in volunteers before and after vitamin $\mathrm{K}$ supplementation, and in patients on vitamin $\mathrm{K}$ antagonist treatment (figure 3)

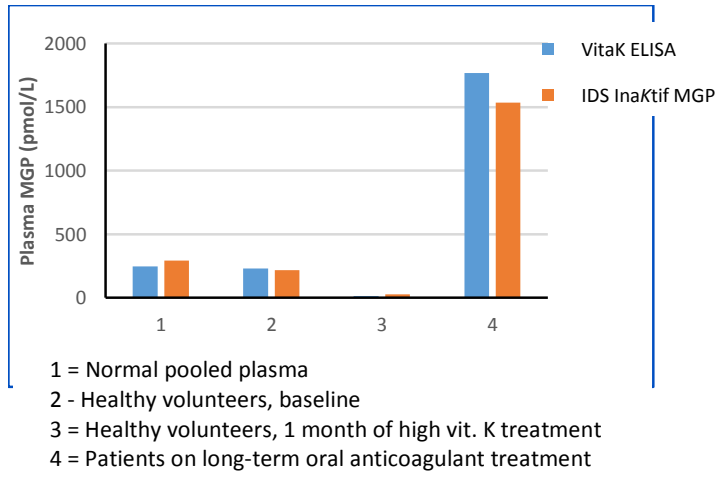

\section{Conclusions}

- $\quad$ The automated dp-ucMGP assay closely resembles the microtiter plate version Therefore, data obtained with both assays are comparable.

- The InaKtif MGP assay provides a quantitative measure for the vitamin K status of the vessel wall.

- Poor vascular vitamin $\mathrm{K}$ status was demonstrated to be a major risk factor for cardiovascular morbidity and mortality, both in patients and in the healthy population

- $\quad$ Studies in which cardiovascular end points are monitored should be stratified for $\mathrm{dp}$ ucMGP.

- $\quad$ Poor vitamin $\mathrm{K}$ status is a modifiable risk factor for arterial calcification, and the InaKtif MGP assay is the most direct way to identify patients at risk and to monitor the effect of vitamin $\mathrm{K}$ supplementation.

\section{References}

Cranenburg, E.C.M., et al. Vitamin K, the coagulation vitamin that became omnipotent. Thromb. Haemostas. 98 (2007) $120-125$.

2. Cranenburg E.C.M., et al. Characterization and potential diagnostic value of circulating matrix Gla protein (MGP) species. Thromb. Haemostas. 104 (2010) 811-822. 